\title{
Recurrent Dedifferentiated Paratesticular Liposarcoma with Synchronous Renal Cell Carcinoma and Prostate Cancer
}

\author{
Coyle D. Flaherty R.A. Kelly B.D. Hynes S.O. \\ Colesky F. Quinlan M.R. Corcoran M.O. Rogers E.
}

Department of Urology, University College Hospital Galway, Newcastle, Galway, Ireland

\section{Key Words}

Liposarcoma $\cdot$ Paratesticular $\cdot$ Sarcoma $\cdot$ Scrotal mass

\begin{abstract}
Paratesticular liposarcoma is a very rare cause of scrotal mass. It is thought that they arise from spermatic cord lipomas most commonly. While well differentiated tumors tend to share many histological similarities with dedifferentiated tumors, the latter has a much more aggressive phenotype. We present an unusual case of a 69-year-old male with synchronous prostate adenocarcinoma and unilateral renal cell carcinoma who was found to have a dedifferentiated paratesticular liposarcoma. Treatment was with radical resection, preserving the testis, followed by radiotherapy. Unusually recurrence did not occur until 4 years following initial treatment. This case demonstrates the high propensity of dedifferentiated liposarcoma to recur locally and examines the most frequently employed management strategies.
\end{abstract}

Copyright $\odot 2012$ S. Karger AG, Basel

\section{Case Report}

A 69-year-old male underwent clinical evaluation and staging investigations for screen-detected high grade prostate adenocarcinoma. A left scrotal mass was discovered on clinical examination. Ultrasound evaluation showed the left testicle to be located in the left inguinal canal, associated with an irregular left sided scrotal mass which was mostly hyperechoic. Serum biochemi-

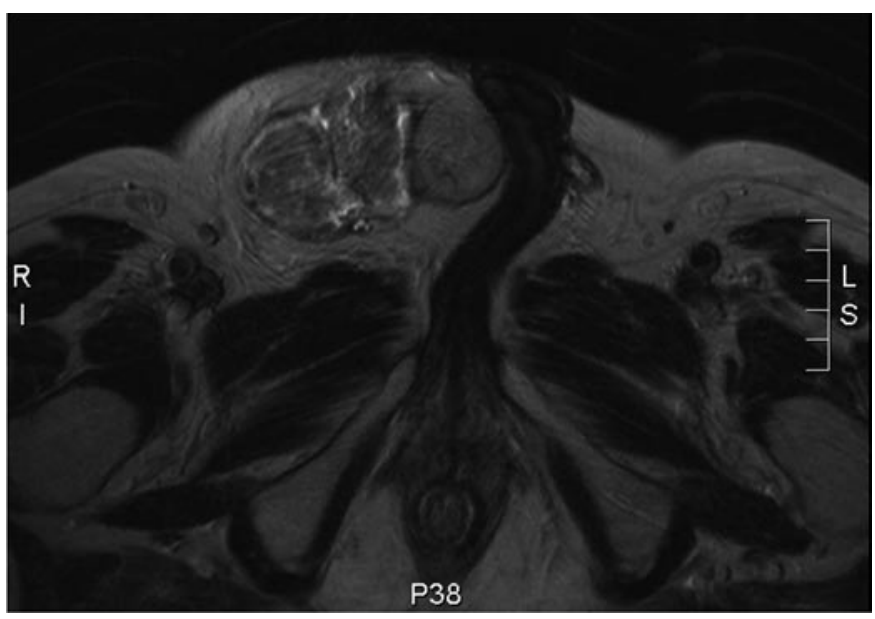

Fig. 1. Magnetic resonance imaging (T1 weighted) of pelvis and scrotum revealing a low-signal intensity mass in the right scrotum with no extension into the retroperitoneum.

Dr. David Coyle

Department of Urology

University College Hospital Galway

Newcastle (Ireland)

E-Mail daithiocool@gmail.com 

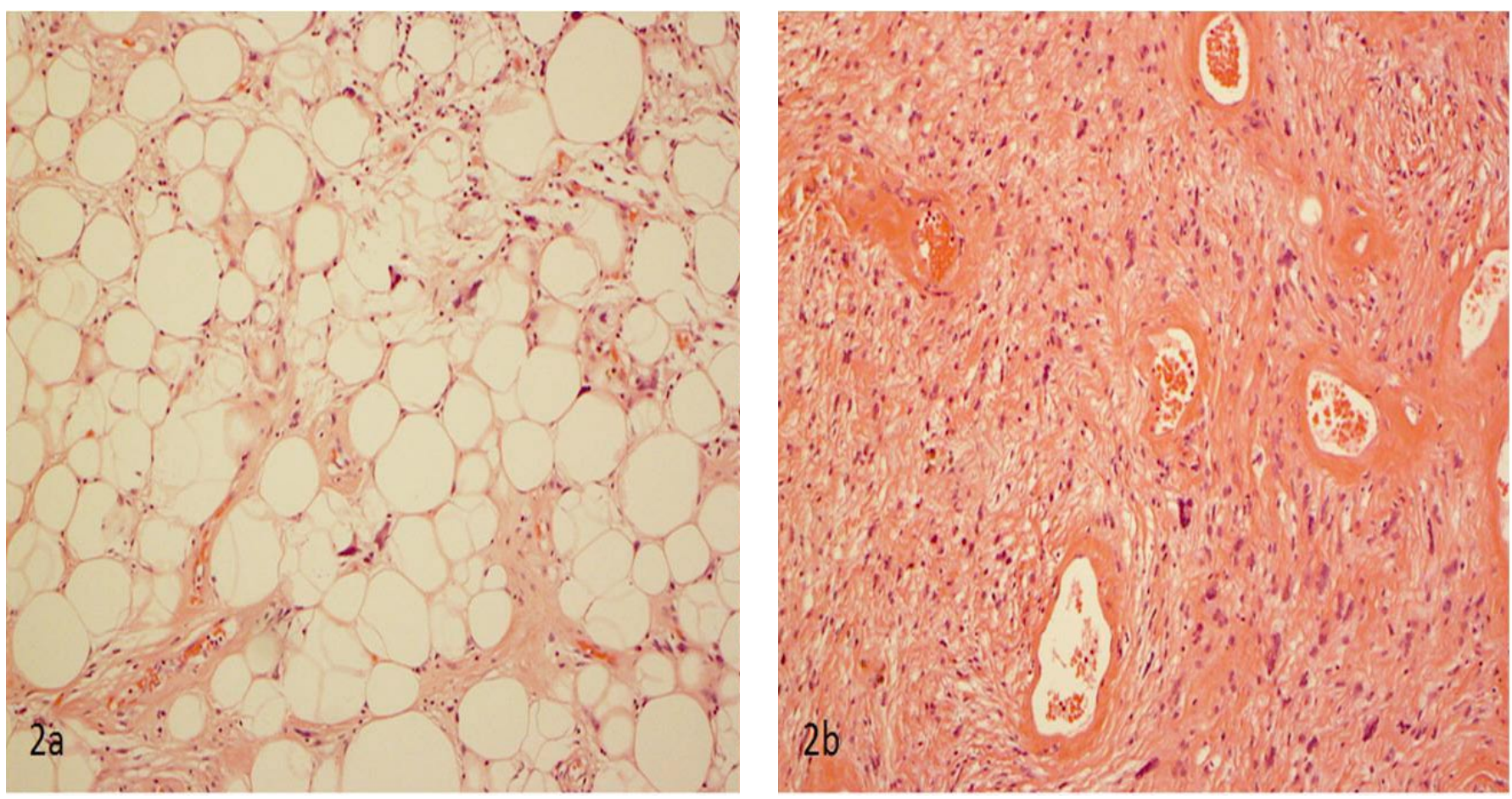

Fig. 2. Histological findings of dedifferentiated liposarcoma with a liposarcomatous elements and $\mathbf{b}$ fibrosarcoma-like cellular sclerotic areas.

cal tumor markers were normal (lactate dehydrogenase $166 \mathrm{U} / \mathrm{l}$; alpha-fetoprotein $3.5 \mathrm{ng} / \mathrm{ml}$; beta human chorionic gonadotropin $<1 \mathrm{IU} / \mathrm{l})$. Magnetic resonance imaging of the abdomen and pelvis demonstrated organ-confined prostate cancer, as well as a $6.5 \mathrm{~cm}$ heterogenous mass at the interpolar region of the left kidney suspicious for renal cell carcinoma with no evidence of lymph node metastasis, and a bulky fatty scrotal mass intimately related to the left testis (fig. 1).

He was commenced on androgen deprivation therapy for prostate cancer. Operative management involved left open radical nephrectomy initially. The renal mass was found to be a $65 \mathrm{~mm}$ clear cell carcinoma invading the perinephric fat (pT3a, Fuhrman grade 2 ). Interval left sided radical resection of the scrotal mass was performed, with the left testicle being preserved. The resection specimen weighed $927 \mathrm{~g}$, comprising the cord and a multicystic fatty tissue mass with white fibrous bands. Histological examination revealed well differentiated fat with lipoblasts separated by sclerotic bands composed of pleomorphic spindle cells, which were invading the capsule and vasculature. Further examination confirmed a dedifferentiated liposarcoma with osteosarcomatous elements. Excision margins contained microscopic disease and adjuvant radiotherapy was undertaken, with the dual purpose of treating his recently diagnosed prostate cancer. Surveillance computed tomography scans at $4,8,12$, and 24 months postoperatively showed no adenopathy or metastasis.

Four years following surgery he re-presented with a new rightsided scrotal mass. Examination revealed a right inguinal hernia and a right scrotal mass extending into the inguinal area. Ultrasound demonstrated a discrete heterogenous vascular lesion inferomedial to the right testis and a markedly abnormal appearing heterogenous right testicle. CT confirmed an $8 \times 5 \mathrm{~cm}$ mass in the right hemiscrotum. Magnetic resonance imaging suggested recurrent paratesticular liposarcoma without extension into the pelvis or perineum.

Right sided radical orchidectomy was performed. Histopathological examination revealed T2b N0 grade 2 dedifferentiated liposarcoma with lipomatous, sclerotic and fibrosarcoma-like areas and without lymphovascular invasion (fig.2). No direct testicular parenchymal invasion was seen. The patient remains under close surveillance for local recurrence and distant metastasis and could be treated with palliative chemotherapy should further resection be technically unfeasible.

\section{Discussion}

Liposarcoma is a malignant neoplasm arising in the mesenchymal precursor cells of adipocytes with a peak incidence in the 5th to 7 th decades of life. It is the second most common type of soft tissue sarcoma after pleomorphic sarcoma, most commonly affecting the retroperitoneum and extremities [1]. 
Paratesticular liposarcoma is rare, and usually arises from malignant transformation of a spermatic cord lipoma, although it may arise from the tunica vaginalis or epididymis. Local retroperitoneal spread with or without distant metastasis is the natural progression of this disease. High tumor grade and elevated lactate dehydrogenase appear to accurately predict disease recurrence and poor outcome [2].

Dedifferentiated liposarcoma appears histologically as well-differentiated liposarcoma but with sharply demarcated segments of non-lipogenic sarcomatous tissue. Despite sharing many histological similarities with well-differentiated liposarcoma, the biological behavior of dedifferentiated liposarcoma is aggressive, with much higher rates of recurrence, metastasis and mortality [1].

Primary surgery traditionally entails radical orchidectomy. Achieving excision margins free of microscopic disease, even when grossly negative, can prove challenging, a difficulty also noted in retroperitoneal liposarcoma, owing mainly to anatomical limitations during resection [3]. Postoperative radiotherapy is often undertaken as lo- cal recurrence rates are high, even in those with completely excised tumor [3]. Consensus from the limited published literature on this topic endorses its role in the adjuvant setting, especially for those who have already had one recurrence [4]. Pelvic irradiation was employed in this case with the dual purpose of adjuvant treatment for the paratesticular liposarcoma and radical treatment of high grade prostate cancer. Chemotherapy, typically with doxorubicin or ifosfamide-based regimens, can be used in the postoperative or palliative settings. Chemotherapy for other types of liposarcoma has yielded mixed results and its use remains controversial [5].

Surveillance for asymptomatic disease recurrence is with a combination of cross-sectional imaging and clinical examination [6]. Recurrences typically occur within 6 months to 2 years of initial resection and grow rapidly with median tumor volume doubling times of approximately 100 days [7]. In this regard it is unusual that in the above case tumor recurrence was not noted until 4 years following the initial presentation.

\section{References}

$>1$ Chung L, Lau SK, Jiang Z, Loera S, Bedel V, Ji J, Weiss LM, Chu PG: Overlapping features between dedifferentiated liposarcoma and undifferentiated high-grade pleomorphic sarcoma. Am J Surg Pathol 2009;33:1594 1600.

$>2$ Korkes F, Castro MG, Romero FR, Godoy G, Amary MF, Fernandes RC, Perez MD: Paratesticular sarcomas in Brazil. Urol Int 2009; 82:448-452.

$>3$ Catton C, Jewett M, O'Sullivan B, Kandel R: Paratesticular sarcoma: failure patterns after definitive local therapy. J Urol 1999;161: 1844-1847.
-4 Littles JF Jr, Matter RC, Herman G: Paratesticular liposarcoma: a report of two cases and review of the literature. J Natl Med Assoc 1992;84:951-955.

$\checkmark 5$ Pervaiz N, Colterjohn N, Farrokhyar F, Tozer R, Figueredo A, Ghert M: A systematic metaanalysis of randomized controlled trials of adjuvant chemotherapy for localized resectable soft-tissue sarcoma. Cancer 2008;113: 573-581.
-6 Montgomery E, Fisher C: Paratesticular liposarcoma: a clinicopathologic study. Am J Surg Pathol 2003;27:40-47.

$>7$ Kim EY, Kim SJ, Choi D, Lee SJ, Kim SH, Lim HK, Song SY: Recurrence of retroperitoneal liposarcoma: imaging findings and growth rates at follow-up CT. AJR Am J Roentgenol 2008;191:1841-1846. 